\title{
A Resonant Damping Study Using Piezoelectric Materials
}

\author{
J. B. Min, K. P. Duffy, B. B. Choi, \\ C. R. Morrison, R. H. Jansen, A. J. Provenza \\ NASA Glenn Research Center \\ Cleveland, Ohio 44135
}

\begin{abstract}
Excessive vibration of turbomachinery blades causes high cycle fatigue (HCF) problems requiring damping treatments to mitigate vibration levels. Based on the technical challenges and requirements learned from previous turbomachinery blade research, a feasibility study of resonant damping control using shunted piezoelectric patches with passive and active control techniques has been conducted on cantilever beam specimens. Test results for the passive damping circuit show that the optimum resistive shunt circuit reduces the third bending resonant vibration by almost $50 \%$, and the optimum inductive circuit reduces the vibration by $90 \%$. In a separate test, active control reduced vibration by approximately $98 \%$.
\end{abstract}

\section{INTRODUCTION}

The requirements for advanced aircraft engine components lead to designs which are more lightweight and efficient, yet more susceptible to excessive vibration, complex dynamic behavior, and uncertain durability and reliability. Smart damping technology could provide a capability to alter the dynamic behavior of a structure intelligently to achieve reduced hazardous vibration and associated dynamic stresses, increased life, and enhanced damage tolerance. Additionally, an optimized integrated application of this technology may provide weight and volume savings over conventional damping technology systems.

Many types of turbomachinery blade damping methods have been investigated in recent years. These include the self-tuning impact damper [1], viscoelastic constrained-layer damping, air-film damping, magneto-mechanical coatings, plasma sprayed coatings, shunted piezoelectric damping, and high-damping shape memory alloys. In addition, some preliminary work has been done with regard to active blade control, including shape memory alloy actuators, active piezoelectric control, actively controlled magnetic bearings, and magneto-rheological fluids. Most of these dampers have yet been used in engines due to the difficulty of implementation and functionality within the extreme engine environment.

Currently at NASA Glenn Research Center (GRC), the development of specialized smart materials, particularly for high temperature and corrosive conditions, are underway. Specifically, the design, development, and application of high temperature piezoelectric have yielded advancement toward the realization of adaptive aerospace structures for NASA missions. Researchers at NASA GRC have been developing new piezoelectric materials that can function at much higher temperatures than most available materials. Currently these materials can function at up to $600^{\circ} \mathrm{C}$, but they are researching materials that can function up to $1000^{\circ} \mathrm{C}$, which should work well within an engine. These and other smart materials can provide significant structural damping performance depending upon their design and application, and further development of smart damping systems using them. These materials will be investigated for both passive damping and active damping devices for the turbomachinery blade application. Candidate smart materials must demonstrate sufficient levels of structural damping over the life of the structure in the expected operating environments. The models developed in this study must be validated through simulation and experimental testing for proof of operation and viability.

The piezoelectric damper solution consists of piezoelectric patches for sensing and actuation which are controlled by an active or passive control circuit. Extensive work has already been performed in this area [2-3]. However, in future we plan to extend these results into higher temperature regimes and under the centrifugal loading experienced in the engine environment. We will investigate using high temperature piezoelectric patches as blade dampers. The piezoelectric damper will be tuned to remove energy at the resonance frequencies, thereby reducing vibration amplitude. It will not be sized to actuate the blade or significantly change the stiffness of the blade.

Piezoelectric materials produce a voltage when deformed and enable them to be used as sensors. This property also enables them to be used as passive vibration dampers since they convert mechanical energy into electrical energy, which can be dissipated in a shunting circuit. Placing shunted piezoelectric patches in appropriate locations on a blade can reduce resonant stresses for a target mode. Piezoelectric materials can also be used for active vibration control as actuators and sensors that can be used to yield damping over a wider frequency range. A schematic sketch of the system is depicted as shown in Figure 1. 


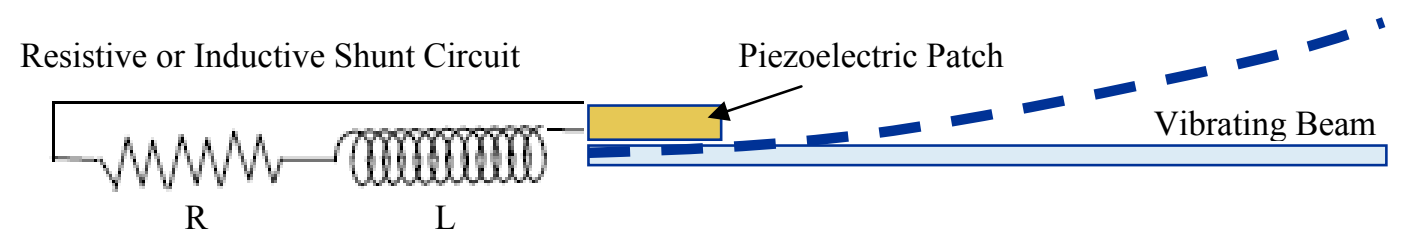

Figure 1: A Sketch of Piezoelectric Patched Beam Specimen with Shunt Circuit

The piezoelectric damper was effective in previous work conducted by various researchers [4-7]. With regards to the turbomachinery application, piezoelectric damping was investigated for stator vanes [8], but there has not been much research into vibration reduction using piezoelectric dampers on turbomachinery rotor blades. Therefore, we have been looking at the rotor blade application to determine how NASA-GRC can improve on the current state of the art in piezoelectric damping. We have started to look at the vibration resonant problem typically causing the HCF problems in the turbomachinery rotor blades.

This study presents some preliminary work done on both passive shunted piezoelectric damping and actively controlled piezoelectric damping for test beams at room temperature.

\section{MATERIALS AND APPROACHES}

The goal of this initial study is to develop vibration reduction techniques for the following target application using shunted piezoelectric materials:

- Fan and/or compressor blades - titanium alloy (e.g. Ti-6Al-4V)

- Maximum temperature of $600^{\circ} \mathrm{F}$

- Harmonic strain amplitudes up to 1000 microstrain

- Thin patch preferred -0.005 " or less

- Target modal damping loss factor $\eta>0.01$ (quality factor $Q<100$ ) vs. undamped loss factor of about $\eta=0.001(\mathrm{Q}=1000)$

- Erosion-resistant and durable.

In this study, cantilever beams with piezoelectric patches were tested with passive shunt circuits and active control to measure vibration reduction versus a baseline undamped beam. Tests were conducted on a vibration exciter (shaker) at room temperature. In future, the work will be extended to show damping effectiveness in plates and blades that exhibit more realistic resonance modes, and at elevated temperatures.

The beam made of Ti-6Al-4V, 8" long x 0.75" wide x 0.092 " thick, clamped at one end was analyzed and tested. The 2nd bending mode was at about $300 \mathrm{~Hz}$, the $3 \mathrm{rd}$ bending mode at about $800 \mathrm{~Hz}$, and the 4th bending mode at about $1500 \mathrm{~Hz}$.

Although we are particularly interested in the 2-stripe mode shown in Figure 2, the mode most likely causing our engine blade HCF problems, we decided to study the $3^{\text {rd }}$ bending mode as an initial start as shown in Figure 3 .

ANSYS [9] finite element models (FEM) with piezoelectric patches were developed for loads that vary sinusoidally with time in the frequency ranges. The frequency response functions (FRF) of the beam tip displacement to the base

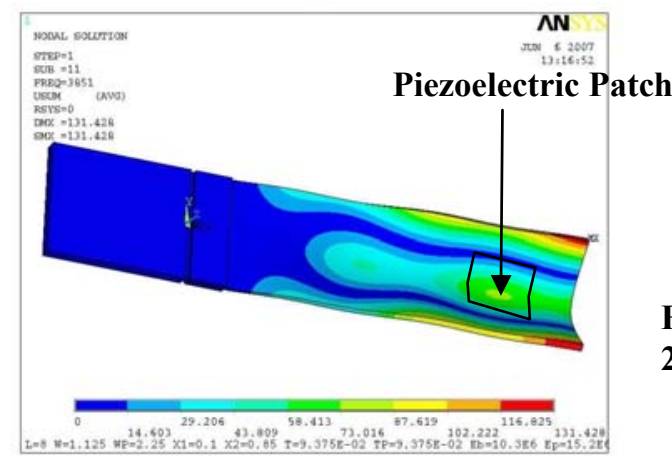

Figure 2: Example Displacement Contour Plot of 2-Stripe Mode Shape for Rectangular Flat Plate Model

acceleration were compared in terms of various resistances $(\mathrm{R})$ and inductances $(\mathrm{L})$ of the passive shunt circuits. The analysis results are validated with the test data and vice versa.

American Institute of Aeronautics and Astronautics

033108 


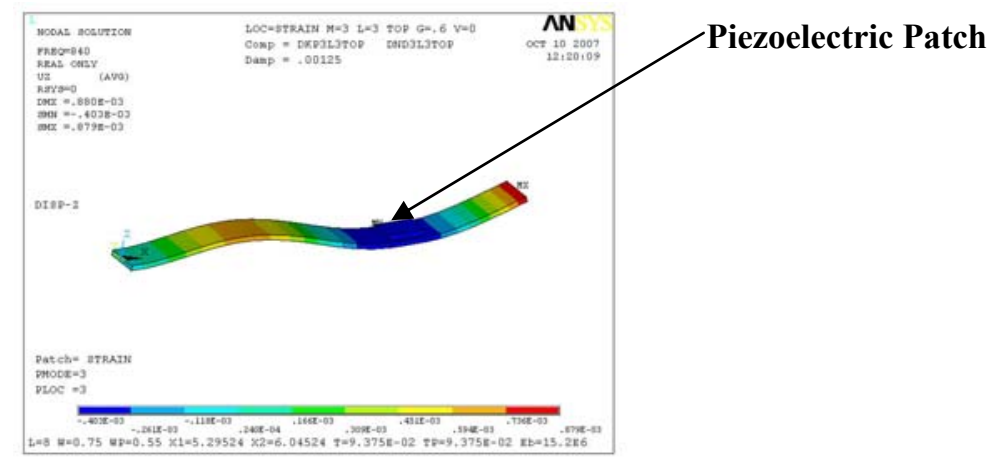

Figure 3: Example Displacement Contour Plot of $3^{\text {rd }}$ Bending Mode Shape for Rectangular Flat Beam Model

Concurrently, parallel efforts have been performed for the active controller development work. The approaches and results obtained from testing and analysis will be described in the following sections.

\section{II.1 CONTROL METHODS AND RESULTS}

Passive techniques can be more easily integrated because of their simplicity and compactness. The piezoelectric elements are connected to a specific electrical network consisting of a dissipative shunt circuit [4]. The most effective method is the tuned shunt where a circuit made with an inductor and a resistor in series is connected to the capacitance of the piezoelectric elements. Optimal damping is obtained by tuning the electrical resonance based on the frequency of the chosen structural mode. Details of the methods employed for our work can be found in the reference [4]. For active control, a controller built with a sensor, a control unit, and a feedback actuator was designed. Additionally, external power sources and amplifiers were used for the control unit and the actuators.

\section{II.1.1 Shunted passive control test procedure and results}

Experiments were performed with the test setup shown in Figure 4. There are two different approaches that were attempted - resistive shunt circuits (resistor only) and inductive shunt circuits (inductor and resistor). These circuits were placed in parallel with a piezoelectric patch placed on the beam in a location of high strain for the third bending mode. A single piezoelectric patch was centered at 5.7" from the base of the cantilever beam, which was clamped onto a shaker. The piezoelectric patch had dimensions of 1.81 " x 0.81 " x 0.010 ". An accelerometer (Endevco model 25A) was located on the clamp and a Bently-Nevada eddy current displacement probe was located at the beam tip. The beam was excited by a 100-lb MB Dynamics vibration exciter. The third bending mode at 835 $\mathrm{Hz}$ was targeted during testing. The piezoelectric material chosen is a Navy Type-II PZT, which has a higher Curie temperature than standard piezoelectric materials.

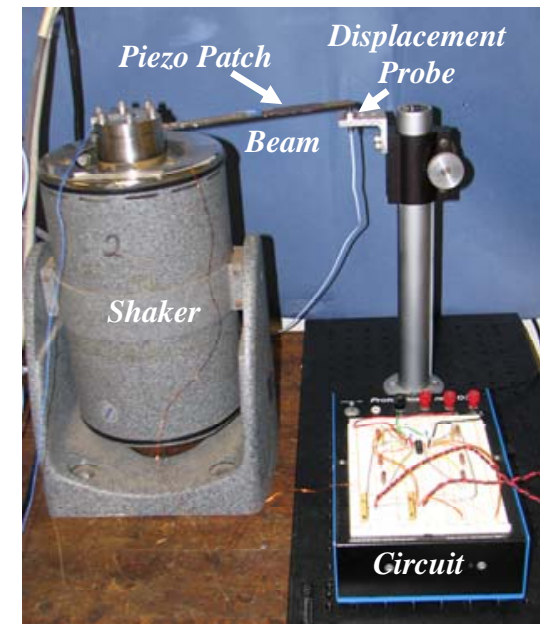

Figure 4: Passive Piezoelectric Damping Test Setup

American Institute of Aeronautics and Astronautics 033108 
Figure 5 shows the base acceleration in G's and the tip displacement in mils versus frequency for the open circuit case. The base acceleration is not constant over the frequency range since the shaker interacts with the beam at resonance.

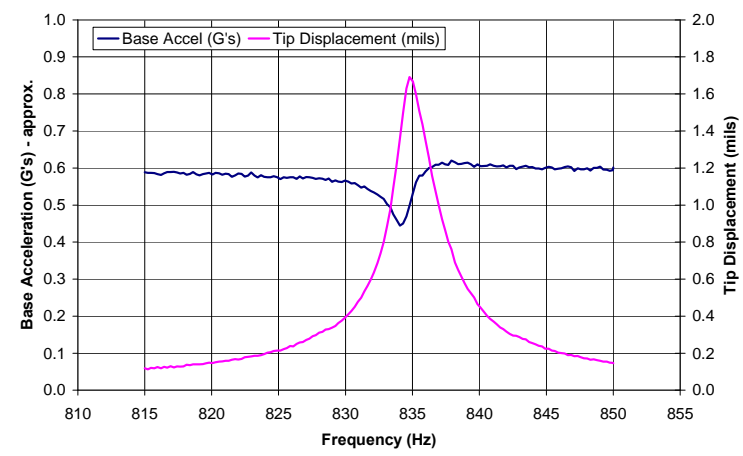

Figure 5: Shaker Base Acceleration and Tip Displacement vs. Frequency

Figure 6 shows the results of placing a variable resistor in parallel with the patch. The damping coefficient was obtained from the transfer function of the tip displacement to the clamp accelerometer. It can be seen that increasing the resistance from 0 to $4000 \Omega$ increases damping - damping more than doubled. The damping decreases above the optimal resistance.

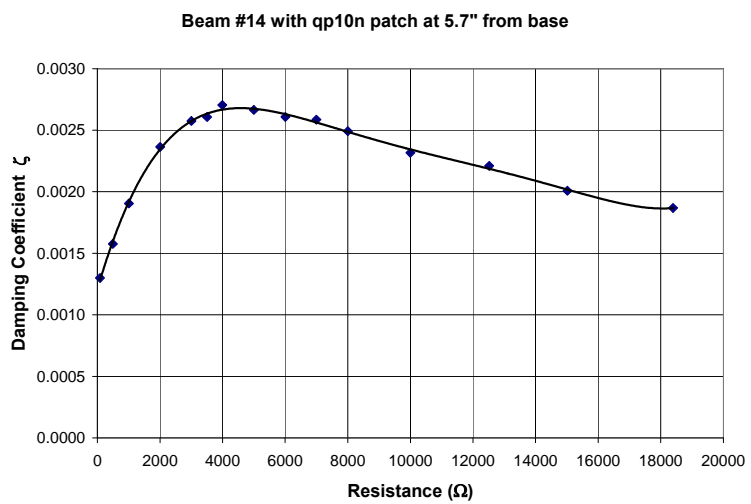

Figure 6: Test Results of Damping Factors with Variable Resistor

The theoretical "equivalent loss factor" [4] of the shunted patch itself is also shown for a resistive-shunted patch as shown in Figure 7. It shows a similar trend to what we can see in the test - lowest damping at zero resistance, then increasing to an optimal value, then decreasing more slowly at higher resistances. The predicted best damping for the current test was $3500 \Omega$ - the test shows the optimum at $4000 \Omega$.

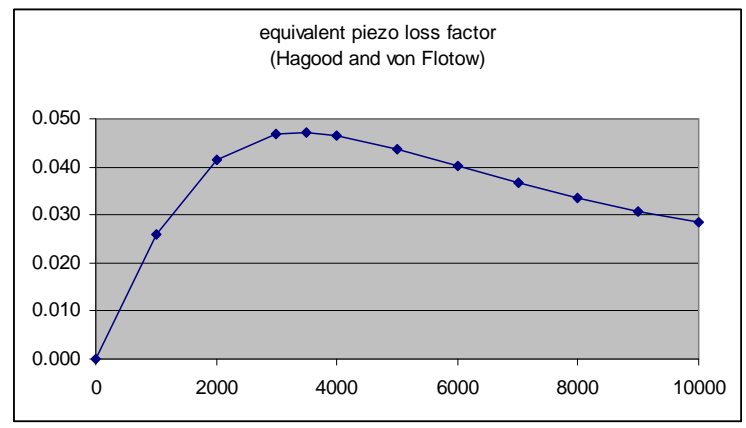

Figure 7: Theoretical Results: Equivalent Loss Factor for Resistor-Shunted Piezoelectric Patch (from Ref. 4) Note: this graph shows patch only loss factor 
A RL circuit attached to the piezoelectric patch was tested. Since the inductance required for this relatively low vibration frequency is rather high, a synthetic inductor circuit was utilized as shown in Figure 4. The circuit that simulates an inductor was based on work by [7]. The inductance of the circuit can be changed with a variable resistor. The RL circuit was tuned to the vibration frequency for an optimal inductance value, which is $0.9 \mathrm{H}$ in this case.

Figure 8 shows how the vibration reduction is affected by the choice of inductance (L) and resistance (R). Test results show that the optimum resistive shunt circuit reduces the resonant vibration by almost $50 \%$, and the optimum inductive circuit reduces the vibration by $90 \%$ for the tuned shunt circuit. For the tuned shunt circuit, the $90 \%$ vibration reduction was only seen for the third bending mode since the circuit targets that particular frequency. The inductor was chosen to match the electrical resonance frequency to the beam vibration frequency. The resistance was then chosen to maximize damping. Without the inductor, there was less vibration reduction. The inductor tunes the shunt circuit to the particular vibration mode of interest, and achieves much better damping.

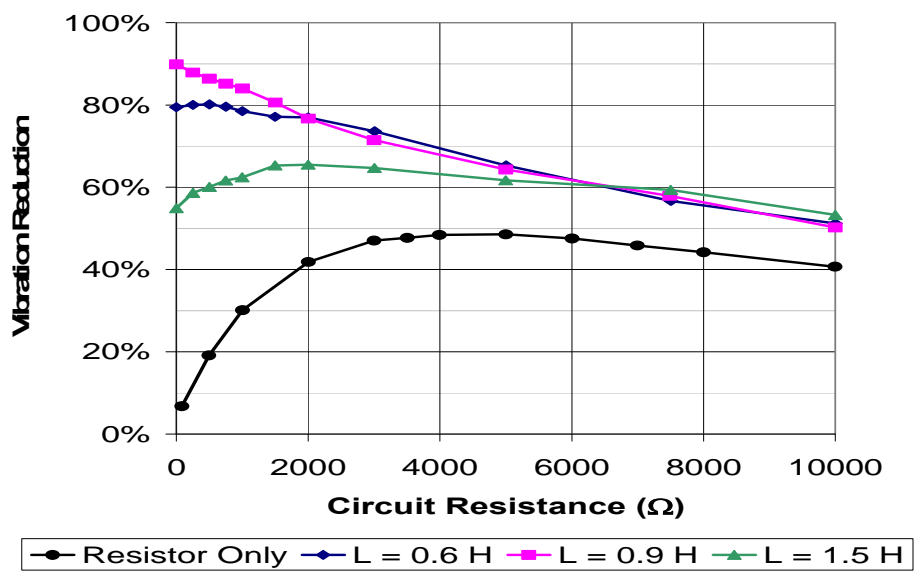

Figure 8: Test Results - Vibration Reduction vs. Shunt Circuit Cases

\section{II.1.2 Shunted active control test procedure and results}

A numerical analysis of a cantilever beam with piezoelectric patches was performed for active control approach using Matlab/Simulink [10] software. A theoretical dynamic model of cantilever beam with piezoelectric patches was derived and then two closed-loop transfer functions were obtained for controller input choice, depending on sensing voltage from patch or displacement probe signal. Based upon the system dynamic model, two control methods (analog shunt circuit and active controlled shunt circuit) were tested numerically. Both controllers worked well. The simulation showed that a resonant controller design using only one pair of piezoelectric patches (actuator and sensor) can be used to damp several resonant modes of the test structure.

Each circuit is tuned to a resonant mode of the piezoelectric patched specimen making the feedback effective only at resonant frequencies [3]. After fine-tuning the controller, the coded control algorithm was downloaded to a dSPACE [11] control system. A controller block diagram is shown in Figure 9.

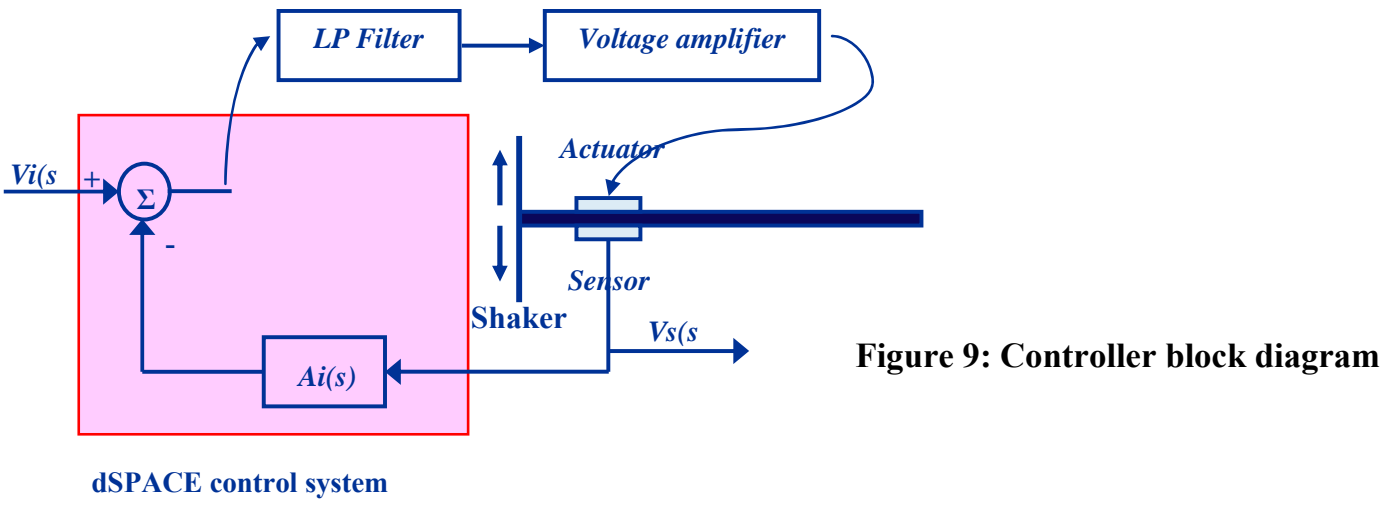

5

American Institute of Aeronautics and Astronautics 033108 
The experimental test setup is shown in Figure 10. A pair of identical piezoelectric patches (sensor and actuator) is bonded at the optimal location for a target resonance - 3rd bending mode. To measure an experimental transfer function of the beam from the shaker (excitation source) to the tip displacement, two accelerometers are attached accordingly. HP Analyzer generates a swept sine signal to send to the shaker and reads all signals from accelerometers, piezoelectric sensor and actuator, and controller voltage from the dSPACE control system. The closed-loop and open-loop transfer functions (Figure 11) were analyzed to investigate damping ratios for the target mode as well as other modes in the operating range.

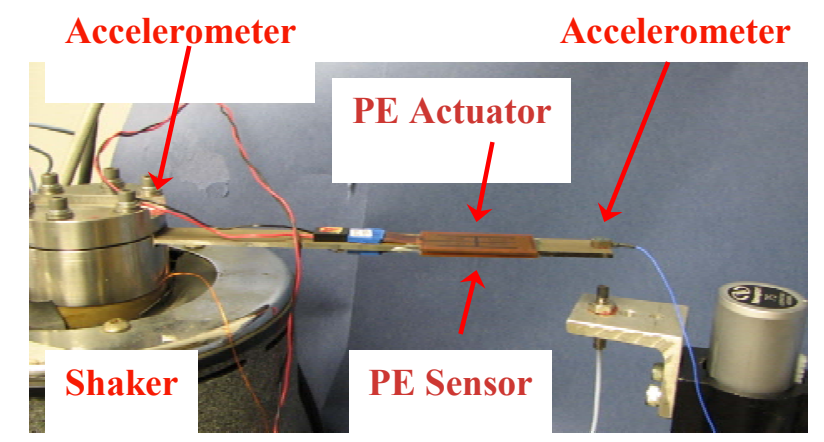

Figure 10: Active Piezoelectric Damping Test Setup

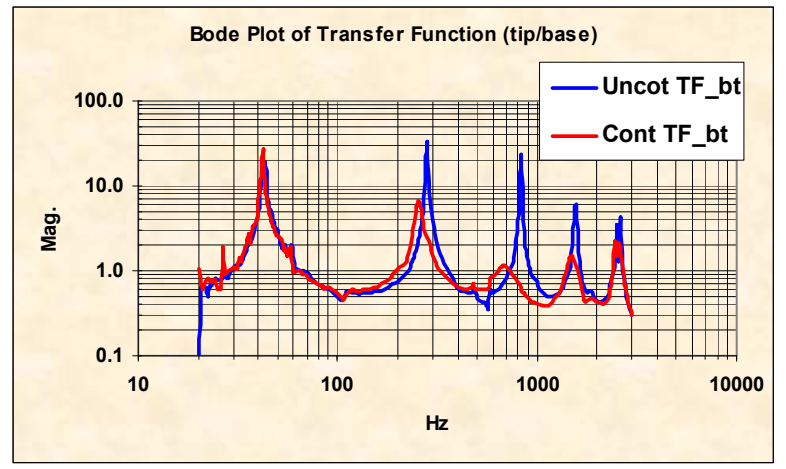

Figure 11: Transfer Functions (tip/base) of Uncontrolled and Controlled Beam with $3^{\text {rd }}$ Mode Targeted from $20 \mathrm{~Hz}$ through $3000 \mathrm{~Hz}$

Reduction of the tip displacement of the $3^{\text {rd }}$ bending mode (controller target) at $824.4 \mathrm{~Hz}$ was achieved by approximately $98 \%$ damping increase by changing a damping factor, $\zeta$, from 0.00371 to 0.141 , and a damping quality factor, Q, from 134 to 3.54 . Reduced neighboring peaks ( $2^{\text {nd }}$ bending and $4^{\text {th }}$ bending modes) by more than $83 \%$ were also observed. It was also shown that higher frequencies such as $5^{\text {th }}$ and $6^{\text {th }}$ modes were affected significantly. However, there was not a much change in the $1^{\text {st }}$ bending mode, which is attributed to the patch location being non-optimal for the first bending mode.

\section{II.2 FINITE ELEMENT MODELING AND RESULTS}

An objective of the finite element modeling (FEM) is to develop an experimentally validated static and dynamic piezoelectric finite element analysis model for the purpose of the piezoelectric controlled structures analysis.

There is a demand for improvement in the modeling tools and very importantly a need for an experimental validation. A vibrating beam damped by the action of piezoelectric elements is considered in this study. A perfect adhesive bonding between actuator and beam is assumed in this model. The beam material was assumed to have a baseline damping level, and the piezoelectric patch had damping that varied according to the shunted damping predicted by Hagood and von Flotow [4]. Shaker acceleration was the input to the finite element model. The output was tip displacement which was subsequently compared to the experimental test results. 
ANSYS software was used to generate the frequency response function (FRF) from a full harmonic analysis. A harmonic analysis study involves two steps. The first step is a modal analysis to determine the natural frequency. The second step consists of a harmonic analysis to give the response over a frequency range.

The model studied was a cantilever titanium beam with a rectangular cross-section. The dimensions and the physical properties are given in Table 1. Two configurations were studied. The first configuration includes a piezoelectric patch bonded onto the only upper face of the beam. In the second case a pair of piezoelectric patches was bonded onto the upper and lower faces of the beam. The geometric and piezoelectric properties of the patch are summarized in Table 2. The piezoelectric patch was centered 5.7 inches from the beam base, a location of high stress for the $3^{\text {rd }}$ bending mode which is the target mode in this study. The analysis tip displacements were compared to the experimental test results in terms of variable damping factors.

Table 1: Ti 6Al-4V beam characteristics

\begin{tabular}{|l|l|}
\hline Length & 8 inch \\
\hline Width & 0.75 inch \\
\hline Thickness & 0.092 inch \\
\hline Young's modulus & $1.52 \mathrm{E}+07 \mathrm{psi}$ \\
\hline Poisson's ratio & 0.29 \\
\hline Density & $0.16 \mathrm{lb} / \mathrm{in}^{3}$ \\
\hline
\end{tabular}

Table 2: Piezoelectric patch characteristics

\begin{tabular}{|l|l|}
\hline Length & 1.81 inch \\
\hline Width & 0.81 inch \\
\hline Thickness & 0.01 inch \\
\hline Location & patch center located at $5.7^{\prime \prime}$ from the beam base \\
\hline Elastic modulus $\left(\mathrm{E}_{11}\right)$ & $6.90 \mathrm{e}+10 \mathrm{~Pa}$ \\
\hline $\mathrm{d}_{33}$ & $3.5 \mathrm{e}-10 \mathrm{~m} / \mathrm{V}$ \\
\hline $\mathrm{d}_{31}$ & $-1.79 \mathrm{e}-10 \mathrm{~m} / \mathrm{V}$ \\
\hline Density & $7700 \mathrm{~kg} / \mathrm{m}^{3}$ \\
\hline
\end{tabular}

As shown in Figure 12, test data showed a tip displacement of 1.668 mils at $834.3 \mathrm{~Hz}$, and a tip displacement of 1.70 mils at $834.5 \mathrm{~Hz}$ was obtained from the FEM for the open-circuit case with a damping factor of 0.001241 . The line in turquoise shown in Figure 12 shows the results obtained from the finite element modeling. The finite element analysis results show a good correlation with the test data.

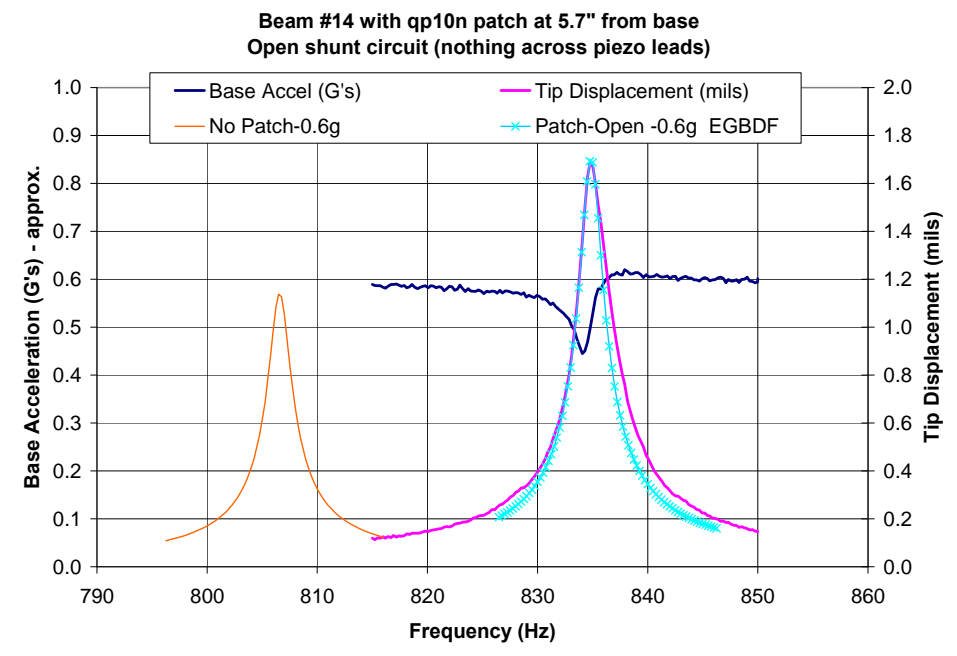

Figure 12: Tip Displacement Comparison: Test Data vs. FEM Results for Open-Circuit

American Institute of Aeronautics and Astronautics 033108 
The open circuit damping was a baseline to investigate the effects of the resistive shunting circuit on the resistive shunted beam system damping. First, the piezoelectric patch-only damping coefficients were calculated using the theoretical equations [4] for different resistance values and applied to the patch elements.

Additionally, a constant beam damping coefficient was applied to the beam elements in the model. The patched beam system damping coefficients were then calculated by the finite element harmonic response analysis using the half-power point method [12]. The beam system damping values calculated by the FEM were compared to the experimental test results and showed a reasonably good correlation as shown in Figure 13. The patched beam system damping values were furthermore evaluated by comparing the beam tip displacement between FEM and experimental test for the resistor-only case as shown in Figure 14. There was a good correlation with the test results.

Following validation of the test results and vice versa, subsequently the same FEM model was further tested for the inductive shunting circuit (RL circuit) analysis. For the inductive shunt circuit modeling, the beam patch system damping values based on the RL shunt circuit experimental test were applied. Here the beam and patch damping values were assumed to be the same. Figure 15 shows a comparison for the inductive shunting results obtained from the experimental test and FEM. It was noticed that a correlation of experimental test and analysis results was practically acceptable, yet somewhat less agreeable as the resistance was increased. Figure16 shows the frequency response functions for open circuit, resistive shunt, and inductive shunt by the finite element model to see the effects of the shunting circuits on the amplitude reductions. Same as with the experimental test results shown in Figure 8, the finite element results also indicate that the inductive shunting can widen and lower the peak resonant amplitude considerably. Without the inductor, there was less amplitude reduction.

\section{Damping Coefficients for Resistive Shunt Test vs. FEM}

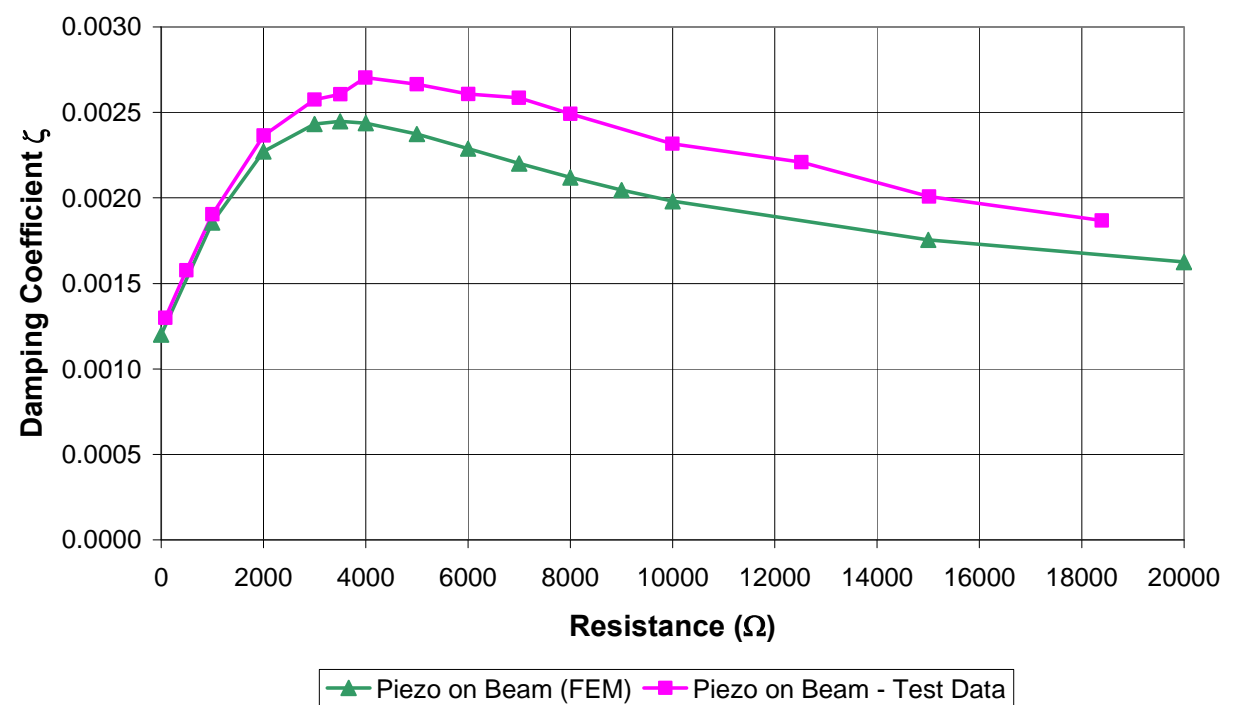

Figure 13: Resistive Shunt Damping Coefficients: Test vs. FEM 


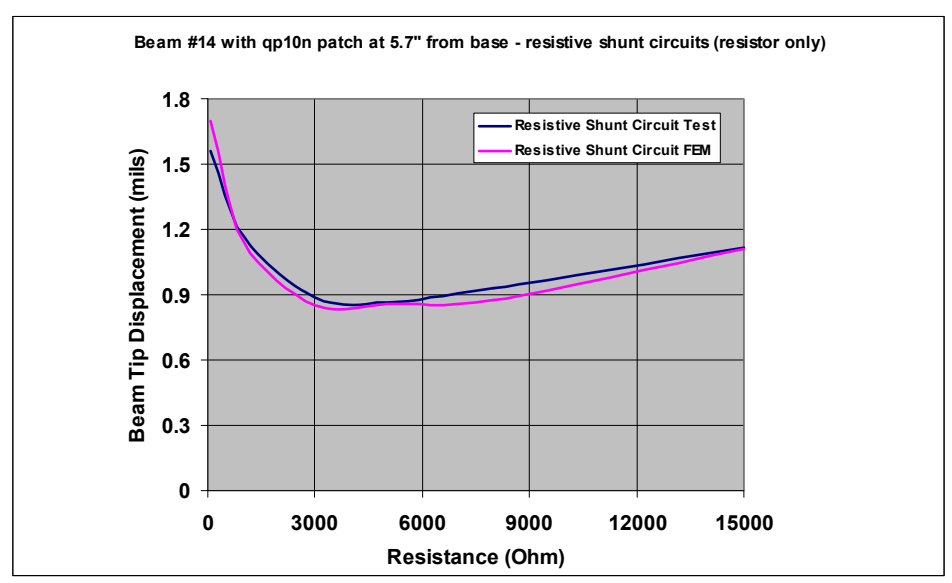

Figure 14: Tip Displacement Comparison for Resistive Shunted Circuit: Test Data vs. FEM Results

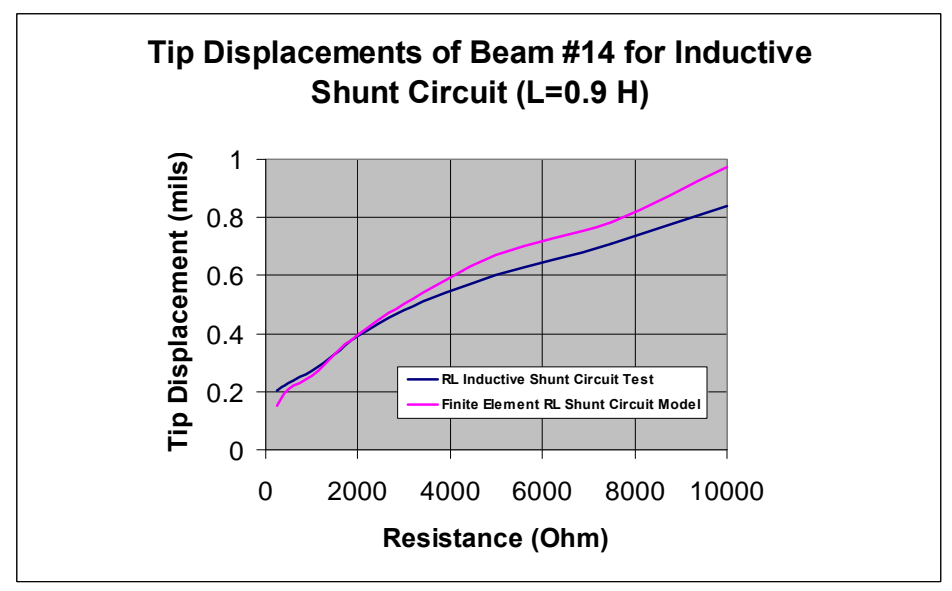

Figure 15: Tip Displacement Comparison for Inductive Shunted Circuit: Test Data vs. FEM Results

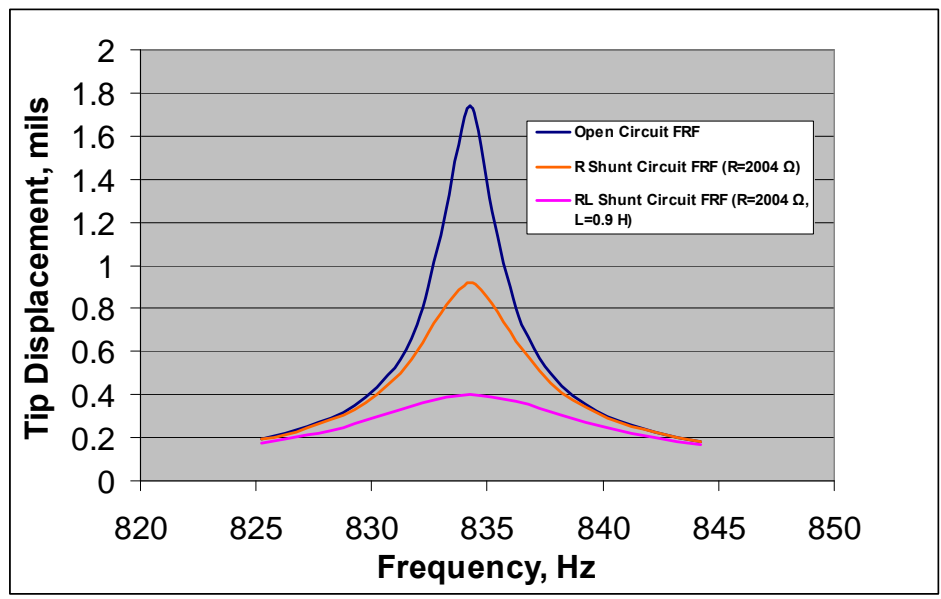

Figure 16: Frequency Response Function (FRF) by FEM for Open Circuit, Resistive Shunt Circuit $(R=2004 \Omega)$, and Inductive Shunt Circuit $(R=2004 \Omega, L=0.9 ~ H)$ 


\section{SUMMARY}

In summary, passive shunted piezoelectric patches were shown to significantly reduce the resonant vibrations of a titanium beam. There are two different approaches that were attempted - resistive shunt circuits (resistor only) and inductive shunt circuits (inductor and resistor). These circuits were placed in parallel with a piezoelectric patch placed on the beam in a location of high strain. The third bending mode at $835 \mathrm{~Hz}$ was targeted during testing. Test results show that the optimum resistive shunt circuit reduces the resonant vibration by almost $50 \%$, and the optimum inductive circuit reduces the vibration by $90 \%$.

For active control technique, a feedback digital controller was used in a separate test to reduce peak amplitudes at the beam specimen resonant modes. Based on the system dynamic model, two control methods (analog shunt circuit and active controlled shunt circuit) were attempted and tested numerically. Both controllers worked well. The simulation showed that a resonant controller design using only one pair of piezoelectric patches (actuator and sensor) can be possible to damp several resonant modes of the test structure. Reduction of the tip displacement of the $3^{\text {rd }}$ bending mode (controller target) was achieved by approximately $98 \%$ damping increase.

The finite element harmonic response analysis in parallel with the resonant damping control tests was utilized to validate the test data and vice versa. There was a reasonably good correlation in the results. Based on the results in a series of the studies, it suggests that the finite element modeling technique developed in this study work for future resonant damping control analysis cases.

\section{WORK IN NEAR FUTURE}

This work is a combination of analytical and experimental study to evaluate the feasibility of passive and active damping approaches to reduce high cycle fatigue problems in turbine engine fan and compressor blades.

Future work will focus on damping vibrations of more complex blade-like modes in flat plates, as well as demonstrating the effectiveness of piezoelectric damping under centrifugal loading in NASA GRC Dynamic Spin Facility. In addition, we will focus on newer high-temperature piezoelectric materials that can function within the engine environment.

Concurrent with testing, analytical work will be done to show the feasibility of the damping approaches, optimize application of each method, and compare to the test data.

\section{REFERENCES}

${ }^{1}$ K. Duffy, G. Brown, and R. Bagley: "Self-tuning impact damper for rotating blades," U.S. Patent 6827551, December 2004

${ }^{2}$ Inderjit Chopra: "Review of State of Art of Smart Structures and Integrated Systems", AIAA JOURNAL, Vol. 40, No. 11, November 2002

${ }^{3}$ Moheimani, S. 2003: "A Survey of Recent Innovations in Vibration Damping and Control Using Shunted Piezoelectric Transducers," IEEE Transaction on Control Systems Technology, 11(4): 482-494

${ }^{4}$ N. W. Hagood and A. von Flotow: "Damping of Structural Vibrations with Piezoelectric Materials and Passive Electrical Networks," J. of Sound and Vibration (1991) 146(2), 243-268.

${ }^{5}$ Davis, C.L., and Lesieutre, G.A.: "A Modal Strain Energy Approach to the Prediction of Resistively Shunted Piezoceramic Damping," Journal of Sound and Vibration, Vol. 184, No.1, pp.129-39, 1995

${ }^{6}$ Wu, S.Y., and Bicos, A.S.: "Structural Vibration Damping Experiments Using Improved Piezoelectric Shunts," Proceedings of the SPIE-The International Society for Optical Engineering, Vol. 3045, pp. 40-50, 1997

${ }^{7}$ Tsai, M. and Wang, K.1999: "On the Structural Damping Characteristics of Active Piezoelectric Actuators with Passive Shunt," Journal of Sound and Vibration, 221(1): 1-22

${ }^{8}$ C. J Cross and S. Fleeter: "Shunted piezoelectrics for passive control of turbomachine blading flow-induced vibrations," Smart Materials and Structures 11 (2002) 239-248

${ }^{9}$ ANSYS Multiphysics code: ANSYS, Inc., Canonsburg, PA, USA

${ }^{10}$ Matlab/Simulink software: The MathWorks, Inc., Natick, MA, USA

${ }^{11}$ dSPACE Systems: dSPACE Inc., Wixom, MI, USA

${ }^{12} \mathrm{~W}$. T. Thomson, Theory of vibration with applications, Prentice-Hall, Inc., 1981 